\title{
Regulatory Defense of the Exercise-Induced Weight Elevation in Hamsters ${ }^{1}$
}

\author{
KATARINA TOMLJENOVIĆ BORER and ALICE A. KOOI
}

\author{
Neuroscience Laboratory, University of Michigan, \\ Ann Arbor, Michigan 48104
}

\begin{abstract}
Twelve adult female hamsters were given free access to horizontal disc exercisers over a 41-day period while 10 hamsters of matching weight remained sedentary. An accelerated weight gain of $1.1 \mathrm{~g} /$ day on Days $7-41$ of activity and during 21 days following its termination led to a $33.8 \mathrm{-g}$ upward displacement of exercising hamsters' weight above the weight level maintained by sedentary controls $(P<0.001)$.

The permanence of the exercise-induced weight elevation was tested by subjecting half of the exercised and half of the sedentary hamsters to a $20 \%$ weight loss. Upon the reintroduction of unlimited food the sedentary and exercised hamsters which had undergone a $20 \%$ weight loss made comparable adjustments in the rate of weight gain and in caloric intake and restored their weight to the level of nonreduced controls. These results demonstrate that disc exercise induces an upward resetting of the regulated weight level in adult hamsters.
\end{abstract}

Adult mammals maintain stable body weight levels over long periods of their life span. Although in the rat weight shifts are induced by absence of food (Widdowson and McCance, 1963), by force feeding (Cohn and Joseph, 1962), by alterations in the levels of gonadal (Tarttelin and Grorski, 1973, Wade, 1972, Zucker, 1969) and adrenal hormones (Mook et al., 1972, Roberts et al., 1972, Stern et al., 1974), and by variable palatability and caloric density of food (Corbit and Stellar, 1964), such weight changes are typically transient. As soon as the hormonal or physiological variables influencing the body weight stop operating, adult rats initiate compensatory behavioral and physiological responses which restore the body weight to that level which prevailed before the onset of weight disturbance. The nature of the regulatory

${ }^{1}$ This research was supported in part by a grant from the Weight Watchers Foundation, Inc. to K. T. Borer. We thank Elliot S. Valenstein for the encouragement and for the use of equipment. Elliot S. Valenstein and J. Roger Wilson made helpful suggestions for the improvement of the manuscript. 
processes responsible for the defense of body weight in adult mammals is at the present time poorly understood (Hoebel, 1971).

In contrast to these temporary weight disturbances Borer (1974) has recently described a manipulation causing a lasting upward weight displacement in adult hamsters. It consists simply of allowing adult hamsters to run on a horizontal activity device. This voluntary exercise leads to sustained acceleration in the rate of weight gain during and for a period of time after disc activity. The weight accumulated in the course of running is maintained during the subsequent 4 - to 6 -wk period. This phenomenon is of considerable interest because it provides a unique opportunity to examine the mechanism of disrupted weight regulation during disc exercise and to further elucidate the process of weight homeostasis in the neurologically and endocrinologically intact animal.

The present experiment was undertaken to establish whether the weight elevation resulting from voluntary exercise in adult female hamsters represents an upward resetting of the regulated weight level. The presence of weight regulation in the retired exercised hamsters was tested by subjecting them to a weight loss equal to the excess weight accumulated in the course of exercise. Weight and food intake of weight-reduced hamsters were monitored for evidence of compensatory responses directed at the restoration of the elevated weight level established in the course of disc exercise.

\section{METHOD}

Subjects. Twenty-two adult female golden hamsters, Mesocricetus auratus (Engle Laboratory Animals, Inc., Farmersburg, IN), weighing about $93 \mathrm{~g}(84-102 \mathrm{~g})$ at the start of the experiment, were used. Animals were matched by weight and assigned to exercising $(n=12)$ or sedentary $(n=10)$ groups at the outset. On Day 82 of the experiment two subgroups of equivalent weight were formed among sedentary hamsters ( $\mathrm{A}$ and $\mathrm{B}, n=5$ each) and exercised hamsters (C and D, $n=6$ each). Groups B and D were subjected to a $20 \%$ weight loss.

Apparatus and procedure. Hamsters were individually housed in suspended cages at all times except for the 41-day period of exercise. During that time period the 12 hamsters in the exercising group were individually housed in Plexiglas boxes ( $30 \mathrm{~cm}$ wide, $40 \mathrm{~cm}$ deep, and $32 \mathrm{~cm}$ high) with permanently mounted horizontal disc exerciser, $25 \mathrm{~cm}$ in diameter (Borer, 1974). Animals were maintained on a $12 \mathrm{~L}: 12 \mathrm{D}$ light schedule and at controlled room temperature of $20-22^{\circ} \mathrm{C}$. Unlimited amounts of water, Purina laboratory rat chow, and sunflower seeds were available at all times with two exceptions: seeds were removed between Days 19 and 40, and between Days 83 and 96 hamsters in groups B and D were limited to about $3 \mathrm{~g}$ of pellets daily in order to reduce their weight by $20 \%$. 
Hamsters were weighed daily. Disc revolutions were recorded on cumulative counters every $24 \mathrm{hr}$ over a 41 -day activity period.

Food intake was measured daily during three discrete periods. The first period of measurement was between Days 1 and 7 , prior to the onset of exercise. Food intake was again measured between Days 75 and 82, at which time the rate of weight gain of exercised hamsters had returned to control levels. Final food intake measurements were taken between Days 97 and 104, $24 \mathrm{hr}$ following the return of unlimited quantities of food to weight-reduced hamsters in groups B and D.

Food intake was measured by subtracting the weight of remaining and spilled pellets and seeds from the weight of food given to hamsters $24 \mathrm{hr}$ earlier. In rare instances in which hamsters were found with food inside their pouches, the food was extracted manually by gentle pressure to the skin surrounding the pouches. Caloric values for Purina laboratory rat chow and sunflower seeds were taken to be 3.61 and $6.83 \mathrm{kcal}$, respectively (Borer, 1974).

To establish the nature of exercise-induced weight increase measurements were made of the body length increments between Days 1 and 142 . The percentage body fat was determined indirectly from the total body water content of hamsters which bears a constant relationship of $73.2 \%$ to the lean body mass in hamster (Kodama, 1971) and in a variety of other mammalian species (Pace and Rathbun, 1945). It is assumed that this relationship does not change during and following exercise-induced weight displacements. Manipulations which affect body fat stores do not alter the water content of lean body mass in rats (Babineau and Pagé, 1955). Body water determinations were made by drying carcasses to constant weight at $60-90^{\circ} \mathrm{C}$.

Duncan's multiple-range test was used to assess the differences between the groups where multiple comparisons were involved. Student's $t$ test was used for comparisons involving two matched groups.

\section{RESULTS}

Activity levels. All animals engaged in vigorous disc activity throughout the period of exposure to horizontal disc exercisers. The mean daily activity level was $26,006 \pm 6,829$ revolutions. Activity levels of individual animals ranged from 12,006 to 36,083 revolutions per day. No correlation existed between the mean activity level and the magnitude of length increment or the magnitude of weight gain (Spearman rank coefficient of correlation $r=-0.18$ and $r=0.39$, respectively).

Weight changes as a function of exercise and diet. Body weight changes of exercising (circles) and sedentary hamsters (triangles) over the course of the experiment are presented in Fig. 1. Upon the introduction of sunflower seeds 


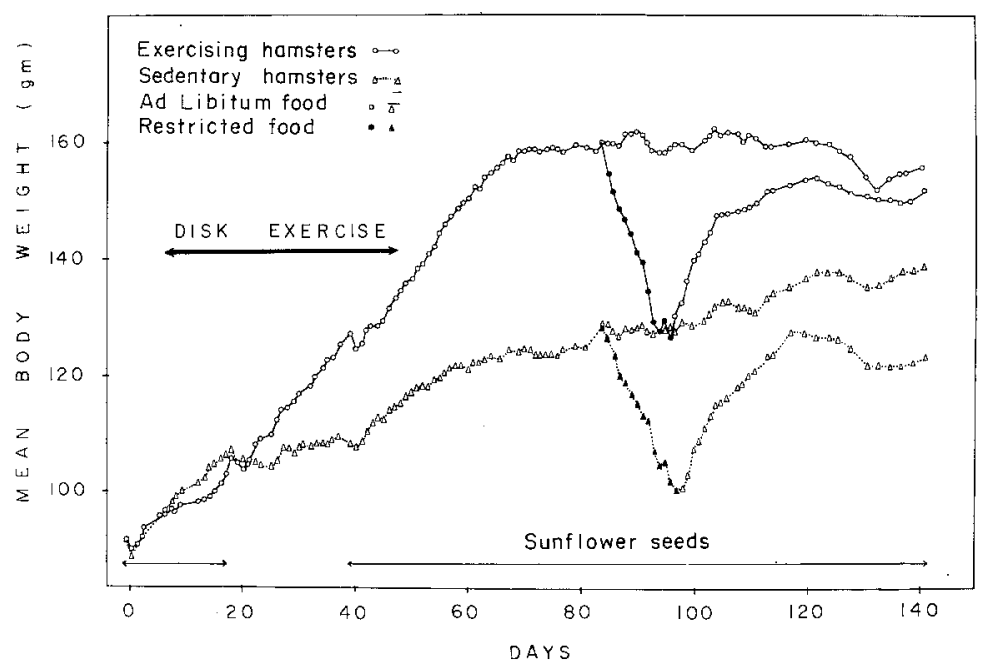

Fig. 1. Body weight changes in sedentary (triangles) and exercising (circles) hamsters as a function of availability of disc exercise, sunflower seeds (horizontal arrows), and during the food restriction to $3 \mathrm{~g}$ of pellets daily (solid symbols).

on Day 1 of the experiment all hamsters showed an initial rapid rate of weight gain of about $0.9 \mathrm{~g} / \mathrm{day}$. The dependence of the initial rapid weight gain on the presence of sunflower seeds was tested by removing the seeds between Days 19 and 40 (interrupted arrow). In the absence of sunflower seeds the rate of weight gain of sedentary hamsters declined to $0.2 \mathrm{~g} /$ day and was restored to $0.7 \mathrm{~g} /$ day upon their reintroduction 21 days later. This seed-dependent acceleration in weight gain was short lived. Within $3 \mathrm{wk}$ of the reintroduction of sunflower seeds the weight gain subsided to $0.2 \mathrm{~g} /$ day and remained at this level throughout the rest of the experiment.

By contrast, after an initial drop in the rate of weight gain to $0.4 \mathrm{~g} / \mathrm{d}$ ay during the first 7 days of activity, the exercising hamsters accumulated $1.1 \mathrm{~g} /$ day over 34 days of exercise and 21 subsequent days of retirement. The removal of sunflower seeds had no effect on the accelerated weight gain in exercising hamsters. The exercise-induced weight acceleration generated a significant weight difference $(P<0.01)$ between the active and sedentary groups by the 26 th day of activity. On the last day of activity the exercising hamsters outweighed the sedentary hamsters by about $18 \mathrm{~g}(P<0.01)$. This weight difference increased to $33.8 \mathrm{~g}(P<0.001) 3 \mathrm{wk}$ after the retirement from discs.

Defense of the exercise-induced weight elevation. A $20 \%$ weight loss in half of the retired exercised hamsters (group D) removed the excess weight accumulated during and after disc activity and returned their weight to the level of sedentary controls (Fig. 1). A comparable weight loss was imposed on 
TABLE 1

Mean Food Intake, in Grams of Pellets and Sunflower Seeds, of Sedentary and Exercised Hamsters During Three 7-Day Periods: (1) Before Disc Exercise (Days 1-7), (2) at the New Elevated Weight Setpoint (Days 75-82), and (3) After a 20\% Weight Loss (Days 97-104)

\begin{tabular}{|c|c|c|c|c|c|c|c|}
\hline \multicolumn{8}{|c|}{ Groups } \\
\hline \multicolumn{4}{|c|}{ Sedentary Hamsters } & \multicolumn{4}{|c|}{ Exercising hamsters } \\
\hline \multicolumn{2}{|c|}{$\begin{array}{c}\mathrm{A}^{a} \\
n=5\end{array}$} & \multicolumn{2}{|c|}{$\begin{array}{c}\mathrm{B}^{b} \\
n=5\end{array}$} & \multicolumn{2}{|c|}{$\begin{array}{c}C^{a} \\
n=6\end{array}$} & \multicolumn{2}{|c|}{$\begin{array}{c}\mathrm{D}^{b} \\
n=6\end{array}$} \\
\hline Seeds & Pellets & Seeds & Pellets & Seeds & Pellets & Seeds & Pellets \\
\hline \multicolumn{8}{|c|}{ Days $1-7$} \\
\hline $3.4 \pm 0.4 c$ & $1.2 \pm 0.8$ & $3.5 \pm 0.5$ & $1.0 \pm 0.3$ & $4.1 \pm 0.3$ & $0.7 \pm 0.2$ & $4.4 \pm 0.8$ & $1.1 \pm 0.6$ \\
\hline \multicolumn{8}{|c|}{ Days $75-82$} \\
\hline $3.7 \pm 0.6$ & $0.6 \pm 0.4$ & $4.0 \pm 0.7$ & $0.4 \pm 0.1$ & $4.3 \pm 0.8$ & $2.3 \pm 0.7$ & $4.4 \pm 1.2$ & $2.1 \pm 1.5$ \\
\hline \multicolumn{8}{|c|}{ Days 97-104 } \\
\hline $3.8 \pm 0.7$ & $0.8 \pm 0.8$ & $5.4 \pm 0.9$ & $0.3 \pm 0.3$ & $3.9 \pm 1.5$ & $2.4 \pm 0.9$ & $5.6 \pm 0.9$ & $2.6 \pm 0.9$ \\
\hline
\end{tabular}

$a_{\mathrm{A}, \mathrm{C}}$ groups of hamsters with unlimited access to food throughout the experiment. $b_{\mathrm{B}}$, D groups of hamsters subjected to food restriction on days 83-96.

${ }^{c}$ Mean \pm standard deviation.

half of the sedentary hamsters (group B). After the return of unlimited amounts of food comparable compensatory increases were seen in the rate of weight gain of both weight-reduced groups. Three weeks after the restoration of ad lib. access to food hamsters in groups B and D recovered all but $5.8 \%$ and $4.5 \%$, respectively, of the weights of their nonreduced controls. The restored weight of groups $B$ and $D$ were not significantly different from the weight of nonreduced groups $A$ and $C$. During the remainder of the experiment groups $\mathrm{B}$ and $\mathrm{D}$ which recovered from a weight deficit maintained a minimum weight difference of $25 \mathrm{~g}(P<0.01)$. Significant weight difference $(P<0.05)$ was also maintained between the nonreduced hamsters in groups $A$ and $\mathrm{C}$ until the last 10 days of the experiment.

Food intake changes. Quantitative food intake data are presented in Table 1 in grams of pellets and seeds consumed and in Fig. 2 in total calories derived from pellets and seeds eaten per $100 \mathrm{~g}$ of hamster body weight. Prior to exercise and at the time the weight of retired hamsters leveled off at the new elevated weight setpoint all hamsters consumed between 4 and $5 \mathrm{~g}$ of seeds and 0.3 and $1.5 \mathrm{~g}$ of pellets daily which amounts to between 21 and 23 $\mathrm{kcal} / 100 \mathrm{~g}$ body weight. Following the restoration of ad lib. food to $20 \%$ underweight hamsters in groups $\mathrm{B}$ and $\mathrm{D}$ their food intake significantly 


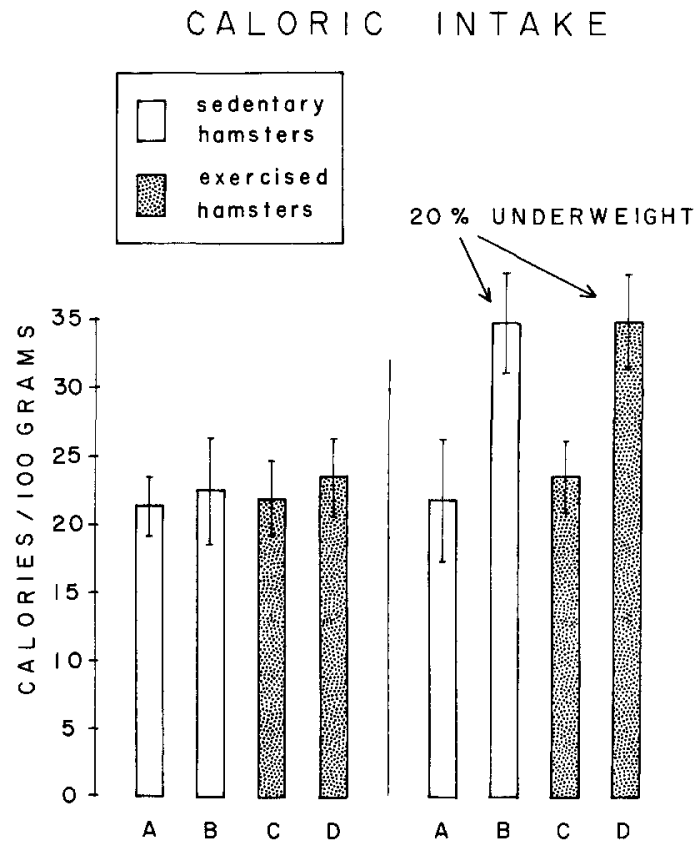

Fig. 2. Mean daily food intake in $\mathrm{kcal} / 100 \mathrm{~g}$ body weight by sedentary (open bars) and exercising (stippled bars) hamsters over a 7-day period before and after the induction of the $20 \%$ weight loss in groups B and D.

increased above that of controls to $5-6 \mathrm{~g}$ of seeds, $0.3-2.6 \mathrm{~g}$ of pellets, or to about $34 \mathrm{kcal} / 100 \mathrm{~g}$ body weight daily $(P<0.001)$.

Preference for sunflower seeds is presented in Fig. 3. The small difference in the sunflower seed preference among the four hamster subgroups during the first week of the experiment was not significant $(P>0.1)$. Average seed preference at that time was about $78 \%$ (left side of Fig. 3). During the second food measurement period 4 wk after the retirement from disc exercisers the exercised hamsters showed a significantly lower seed preference than the sedentary hamsters $(66.7 \pm 15.4 \%$ vs $88.5 \pm 4.8 \%$, respectively, $P<0.05$, middle part of Fig. 3).

Unlike disc activity, the $20 \%$ weight loss did not affect dietary selection (right side of Fig. 3) despite its large effect on quantity of food consumed (Fig. 2, Table 1). The underweight refed hamsters maintained the seed preferences established during the preceding activity manipulation, that is the sedentary hamsters consumed a significantly larger proportion of seeds in their diet $(94.0 \pm 4.6 \%)$ than the exercised hamsters $(67.2 \pm 11.9 \%, P<0.01)$.

Body composition changes. Table 2 lists the body length increments of exercised and sedentary hamsters over the duration of the experiment and the 


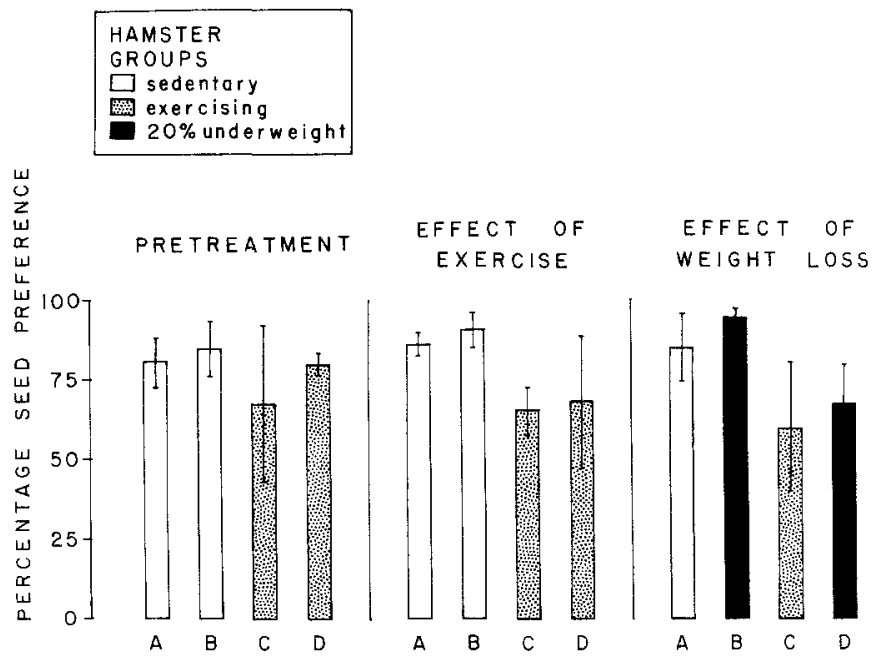

Fig. 3. Mean daily intake of sunflower seeds as a percentage of total weight of food ingested daily by sedentary hamsters (open bars) and by exercising hamsters (stippled bars) during three measurement periods: (1) during the first premanipulation week, (2) $4 \mathrm{wk}$ after the retirement from discs, and (3) following a $20 \%$ weight loss by sedentary group B and retired group D (solid bars).

TABLE 2

Body Length Increments and Percentage Body Fat of Hamsters as a Function of Disc Exercise and of a 20\% Weight Loss ${ }^{a}$

\begin{tabular}{|c|c|c|c|c|}
\hline & \multicolumn{2}{|c|}{ Exercised hamsters } & \multicolumn{2}{|c|}{ Sedentary hamsters } \\
\hline \multirow{2}{*}{$\begin{array}{l}\text { Body length } \\
\text { increment }(\mathrm{cm})\end{array}$} & \multicolumn{2}{|c|}{$\begin{array}{l}n=12(154.8 \mathrm{~g}) \\
1.76 \pm 0.49\end{array}$} & \multicolumn{2}{|c|}{$\begin{array}{l}n=10(131.4 \mathrm{~g}) \\
1.35 \pm 0.20\end{array}$} \\
\hline & Control & Underweight & Control & Underweight \\
\hline Percentage body fat & $\begin{array}{l}n=6(157.2 \mathrm{~g}) \\
21.13 \pm 5.96\end{array}$ & $\begin{array}{l}n=6(152.3 \mathrm{~g}) \\
20.98 \pm 5.3\end{array}$ & $\begin{array}{l}n=5(139.2 \mathrm{~g}) \\
21.4 \pm 3.39\end{array}$ & $\begin{array}{l}n=5(123.6 \mathrm{~g}) \\
19.4 \pm 1.65\end{array}$ \\
\hline
\end{tabular}

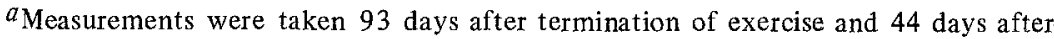
termination of food restriction. Numbers in parentheses indicate the mean body weight of hamster groups at the time of measurements.

final percentage body fat of all the animals. The mean length increment of $1.76 \mathrm{~cm}$ in the retired exercised hamsters significantly exceeded the $1.35-\mathrm{cm}$ length increment of sedentary hamsters $(P<0.05)$. Five of the retired animals have grown more than $2 \mathrm{~cm}$ in length during the 142 days of the experiment, 
while the greatest length among the sedentary hamsters was $1.7 \mathrm{~cm}$ in a single animal.

The percentage of body fat of all hamsters at the end of the experiment was comparable (19.4-21.4\%) despite their different history of activity and weight reduction manipulations.

\section{DISCUSSION}

Voluntary exercise on horizontal discs induces accelerated weight gain and upward weight displacements in adult golden hamsters. In the present experiment we have examined the permanence of the exercise-induced weight elevation. First, we have shown that the elevated weight of the retired hamsters persists when the period of observation is extended from 34 days (Borer, 1974) to 94 days. Furthermore, when the elevated weight level is challenged with a weight loss commensurate to the excess weight accumulated during exercise, the retired hamsters defend their elevated weight as vigorously as do sedentary hamsters subjected to a comparable weight loss. Following a $20 \%$ weight loss the retired hamsters restore their weight to the elevated setpoint as rapidly and as precisely as the sedentary hamsters. While regaining weight the retired and sedentary hamsters show comparable increases in caloric consumption. We, therefore, conclude that voluntary disc exercise leads to upward resetting of the regulated weight level in adult hamsters. Some concomittant of voluntary disc activity interferes with the regulatory processes maintaining adult weight level.

Since disc exercise induces an increase in body length and proportional increases in total body mass and percentage body fat, we propose that suppression of growth be considered as a possible mechanism of weight regulation in adult mammals. It is known that rats retain the potential for skeletal growth (Donaldson and Conrow, 1919) and for elongation of long bones (Dawson, 1925) throughout much of their adult lifetime. The rates of skeletal growth are, however, very low in adult rats. Yet the ability to undergo rapid growth in length can be seen in adult rats. For instance, the sexually mature adult female rat is smaller and shorter than is the male of comparable age (Schemmel et al., 1969). Ovariectomy leads to accelerated weight gain and to a permanent upward displacement of body weight (Mook et al., 1972, Tarttelin and Gorski, 1973). This weight increase is the result of growth in length (Kakolewski et al., 1968) as well as of increases in lean body mass and in the size of most major body compartments (Leshner and Collier, 1973). Active suppression of growth, therefore, may be a participant in the weight-regulatory mechanism in rat as well as in hamster. The major apparent difference between the two species is that gonadal hormones participate in the weight regulation in adult rats but have little influence over body weight in 
adult hamsters (Zucker et al., 1972). In the same way, the integrity of gonads is of little consequence for the appearance of exercise-induced weight gain in adult hamsters (Borer and Powers, in preparation) even though there is an apparent sex difference in the magnitude of induced weight change in exercising hamsters (Borer, 1974, Borer and Powers, in preparation).

In the present experiment we failed to confirm that disc activity induces an accumulation of excess body fat in hamsters with access to both pellets and sunflower seeds (Borer, 1974). In both the previous and the present study disc activity was found to be associated with significantly greater final body length (Borer, 1974) and length increments than the body lengths and length increments observed in sedentary animals. This suggests that the primary effect of disc exercise is to stimulate accelerated growth in length. The discrepancy in the status of the body fat in retired hamsters with access to pellets and seeds in the two studies may be related to different time intervals employed between the termination of exercise and the fat determination. We hypothesize that growth in length occurs during the exposure to disc activity and that accumulation of fat takes place immediately following termination of exercise while retired hamsters persist in their high caloric intake and gain weight at an excessive rate (Borer, 1974). Furthermore, we hypothesize that this retirement fat accumulation is dissipated over the period of time until the body fat content corresponds to the new increased body size. A modest weight loss followed the exaggerated weight gain in retired hamsters eating pellets (Borer, 1974). Hamsters given pellets and sunflower seeds may be unable to dissipate this excess fat as rapidly in view of the high preference for seeds and the high fat content of seeds. Thus, body fat determinations 1 mo after the termination of exercise would yield high body fat values (Borer, 1974) while the body fat measurements 3 mo after the retirement from discs (present study) would reveal body fat content appropriate for the new elevated weight level.

A change in the dietary selection was again found to be associated with the prior exposure to disc exercise. Possibly due to the longer disc exposure in this study the reduced seed preference was found to persist throughout the two measurement periods in hamsters which have reached their new elevated weight level. This exercise-altered dietary selection persisted even in face of hyperphagia exhibited by hamsters compensating for a $20 \%$ weight loss. It will be of interest to determine whether this lasting change in dietary selection results from altered endocrine status or is related to some experiential factor.

In summary, the accelerated weight gain observed when the adult hamsters freely exercise on horizontal discs, represents an upward resetting of their regulated weight level. This weight displacement is associated with growth in length and with the preservation of constant body composition. It is proposed that weight regulation in the adult hamster, and possibly in adult mammals in general, be viewed as a process of controlled suppression of 
growth. The phenomenon of upward weight resetting available through the simple manipulation of voluntary disc exercise offers a valuable opportunity to search for the agent responsible for disrupted weight regulation and for the inappropriate spurt of growth in the intact adult mammal.

\section{REFERENCES}

Babineau, L.-M., and Pagé, E. (1955). On body fat and body water in rats. Can. $J$. Biochem. Physiol. 33, 970-979.

Borer, K. T. (1974). Absence of weight regulation in exercising hamsters. Physiol. Behav. 12, 589-597.

Cohn, C., and Joseph, D. (1962). Influence of body weight and body fat on appetite of "normal" lean and obese rats. Yale J. Biol. Med. 34, 598-607.

Corbit, J. D., and Stellar, E. (1964). Palatability, food intake and obesity in normal and hyperphagic rats. J. Comp. Physiol. Psychol. 58, 63-67.

Dawson, A. B. (1925). The persistence of the cartilage plates in the long bones of the white rat. Anat. Rec. 27, 202 (Abstract).

Donaldson, H. H., and Conrow, S. B. (1919). Quantitative studies on the growth of the skeleton of the albino rat. Amer. J. Anat. 26, 237-314.

Hoebel, B. G. (1971). Feeding: neural control of intake. Annu. Rev. Physiol. 33, 533-568.

Kakolewski, J. W., Cox, V. C., and Valenstein, E. S. (1968). Sex differences in body weight change following gonadectomy in rats. Psychol. Rep. 22, 547-554.

Kodama, A. M. (1971). In vivo and in vitro determinations of body fat and body water in the hamster. J. Appl. Physiol. 31, 218-222.

Leshner, A. I., and Collier, G. (1973). The effects of gonadectomy on the sex differences in dietary self-selection patterns and carcass composition of rats. Physiol. Behav. 11, 671-676.

Mook, D. G., Kenney, N. J., Roberts, S., Nussbaum, A. I., and Rodier, W. I., III (1972). Ovarian-adrenal interactions in regulation of body weight by female rats. J. Comp. Physiol. Psychol. 81, 198-211.

Roberts, S., Kenney, N. J., and Mook, D. G. (1972). Overeating induced by progesterone in the ovariectomized, adrenalectomized rat. Horm. Behav. 3, 267-276.

Pace, N., and Rathbun, E. N. (1945). Studies on body composition. III. The body water and chemically combined nitrogen content in relation to fat content. J. Biol. Chem. 158, 685-691.

Schemmel, R., Mickelsen, O., and Tolgay, Z. (1969). Dietary obesity in rats: influence of diet, weight, age, and sex on body composition. Amer. J. Physiol. 216, 373-379.

Stern, J. J., Porterfield, A. L., and Krupa, R. J. (1974). Endocrine interactions in regulation of body weight in female rats. J. Comp. Physiol. Psychol. 86, 926-929.

Tarttelin, M. F., and Gorski, R. A. (1973). The effects of ovarian steroids on food and water intake and body weight in the female rat. Acta Endocrinol. 72, 551-568.

Wade, G. N. (1972). Gonadal hormones and behavioral regulation of body weight. Physiol. Behav. 8, 523-534.

Widdowson, E. M., and McCance, R. A. (1963). The effect of finite periods of undernutrition at different ages on the composition and subsequent development of the rat. Proc. Royal Soc. Ser. B 158, 329-342.

Zucker, I. (1969). Hormonal determinants of sex differences in saccharine preference, food intake and body weight. Physiol. Behav. 4, 595-602.

Zucker, I., Wade, G. N., and Ziegler, R. (1972). Sexual and hormonal influences on eating, taste preferences and body weight of hamsters. Physiol. Behav. 8, 101-112. 